\title{
Suspended conductive plate oscillations in the magnetic field of the conductor with alternating current
}

\author{
Ivan Popov ${ }^{1}$, Alexey Lukin ${ }^{1}$, Dmitry Skubov ${ }^{1,2, a}$ and Lev Shtukin ${ }^{1,2}$ \\ ${ }^{1}$ St. Petersburg State Polytechnical University, Mechanics and Control Processes Department \\ 2 Institute of Problems of Mechanical Engineering RAS
}

\begin{abstract}
The problem of cooling the conductor with an alternating high-ampere electric current is offered to be solved by using oscillations of suspended conductive plate. System basic parameters are estimated from analysing the system of differential equations describing the motions in coupled electrical-mechanical system. The parameters must satisfy the conditions of system's resonance. Examination of equilibrium position causes a researching of the differential equation with periodic coefficients.
\end{abstract}

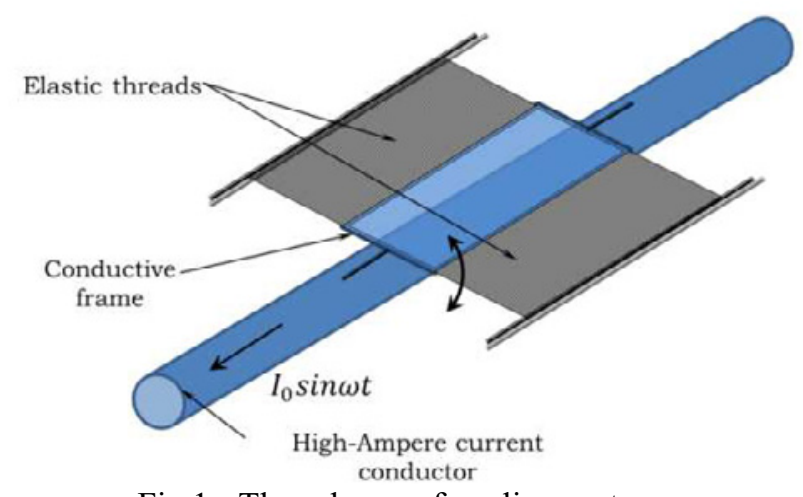

Fig.1 - The scheme of cooling system

The purpose of this work is the research of the autonomous cooling system of the high-ampere conductor with an alternating electric current. The system is supposed to be autonomous in the sense that neither initiation of oscillations, nor circulation of air shouldn't demand additional expenses in the form of power supplies or motive devices. The scheme of this system is given in Fig.1

For analytic efficiency evaluation of suggested system the high-ampere conductor is considered as the infinite long copper cylinder. The suspended plate is located parallel to an axial axis of conductor. The plate is wide and long to generate sufficient cooling airflow. The plate suspended on vertical elastic threads, attached to the middle top and bottom (or left and right) the plate parties. The plate is made of insulator and bordered by the conductive copper frame. The alternating magnet flux, crossing over the conductive frame, is produced by the flow of alternating current in straight conductor. Current variability $(50 \mathrm{~Hz})$ inducts internal EMF in the conductive frame, and this causes the alternating inducted current inside the frame. The amount of generating torque depends on time in sufficiently complex way, by virtue of the fact that under frame rotation the magnet flux changes and therefore the inducted EMF and the current in the frame, as well as the amount of the magnet induction, crossing over its parallel sides. The cross section of the conductor and the conductive frame is given in Fig. 2 .

The genesis of torque is the interaction of the inducted frame currents and the external field induction (in the opposite frame sides inducted currents are oppositely directed and periodically varied by the changing of the magnet flux).

\footnotetext{
$\overline{{ }^{a} \text { Corresponding author: skubov.dsk } @ y a n d e x . r u ~}$
} 


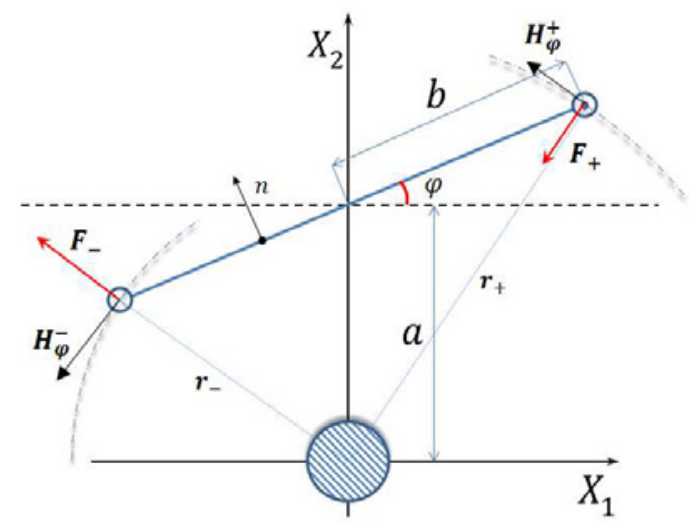

Fig. 2 - Cross section of the conductor and the conductive frame

In a rigorous formulation the magnetic flux as well as inducted currents (and, as a result, the torque) depend not on only directly on time but also on the rotation angle. Therefore the mechanical and electrical current oscillations for the frame are interrelated.

In general these oscillations should be found coupled, as a system of two differential equations with the following determination of the largest amplitude of rotary oscillations, using possible variations of parameters, including the resonant oscillations setting.

For getting the periodical process, it's necessary to consider the nonlinear behavior of the coupled electricalmechanical equations on account of the interaction of electrical and mechanical processes not only linear resonances, but also parametric resonances appear.

Satisfying parametric resonance conditions for rotary oscillations the estimation of the parameters could be found, instead of model of the plate with the conductive frame, but for the case of using plane thin conductive plate, for example, of aluminum. Let's put mathematical model of such a system and make the assumptions about it to simplify analytical computations:

Current flow in cylindrical conductor

$$
I_{0}(t)=I_{0} \sin v t \text {. }
$$

The magnetic field's intensity of the conductor is determined by a simple formula

$$
\underline{H}=H_{\varphi} \underline{e}_{\varphi}=\frac{I_{0}(t)}{2 \pi r} \underline{e}_{\varphi}
$$

Ponderomotive forces, applied on conductor's linear unit and inducted EMF, are described by Ampere's and Faraday's laws [4]

$$
\begin{gathered}
d \underline{F}=\mu \mu_{0} i d \underline{l} \times \underline{H} . \\
E=-\frac{\partial \Phi}{\partial t} .
\end{gathered}
$$

The external magnetic field flux crossing over the frame

$$
\Phi=\int_{(S)} \underline{B} \cdot d \underline{S}=\mu \mu_{0} l \int_{(S)} \underline{H} \cdot \underline{n} d s
$$

Integrating the (5) we use the form of the magnet intensity by means of the magnetic potential in cylindrical coordinate system:

$$
\underline{H}=\operatorname{rot} \underline{A}, \quad \underline{A}=\left\{\begin{array}{lll}
0 & 0 & -\frac{I_{0}(t)}{2 \pi} \ln r
\end{array}\right\}^{T}
$$

Substitution in (5), using the Stokes' theorem [1]

$$
\begin{aligned}
& \Phi=\int_{(s)} \operatorname{rot} \underline{A} \cdot \underline{n} d s=\oint_{(l)} \underline{A} \cdot d \underline{l}= \\
& -\mu \mu_{0} l \frac{I_{0}}{2 \pi} \sin v t \ln \left[\frac{r_{+}(\varphi)}{r_{-}(\varphi)}\right]
\end{aligned}
$$

Where $r_{-}$and $r_{+}$(Fig.2) are

$$
\begin{aligned}
& r_{+}^{2}(\varphi)=a^{2}+b^{2}+2 a b \sin \varphi \\
& r_{-}^{2}(\varphi)=a^{2}+b^{2}-2 a b \sin \varphi
\end{aligned}
$$
the frame:

Electromagnetic forces exert on opposite sides of

$$
\begin{aligned}
& \underline{F}_{-}=\mu \mu_{0} i l\left(H_{\varphi-}\right) \underline{e}_{r-}, \\
& \underline{F}_{+}=-\mu \mu_{0} i l\left(H_{\varphi+}\right) \underline{e}_{r+},
\end{aligned}
$$

where $\underline{e}_{r-}, \underline{e}_{r+}-$ radial orts in appropriate points

The torque can be found

$$
\begin{gathered}
M=-\mu \mu_{0} i l b\left(\frac{H_{\varphi+}}{r_{+}}+\frac{H_{\varphi-}}{r_{-}}\right) \operatorname{acos} \varphi= \\
\mu \mu_{0} i I_{0} \operatorname{sinvt} l \frac{a b}{2 \pi}\left(\frac{1}{r_{+}^{2}}+\frac{1}{r_{-}^{2}}\right) \cos \varphi,
\end{gathered}
$$

or finally

$$
M=-\mu \mu_{0} \frac{a b l\left(a^{2}+b^{2}\right)}{\pi\left[\left(a^{2}+b^{2}\right)^{2}-4 a^{2} b^{2} \sin ^{2} \varphi\right]} I_{0}(t) i
$$

For the frame of length $l$ inertia momentum can be written

$$
J=\left(\frac{4}{3} \rho_{1} b^{3} \delta+2 \rho_{1} b^{2} \delta l+\frac{2}{3} \rho_{2} b^{3} l\right) h,
$$

where $\rho_{i}, i=1,2$ - density of the frame materials (Fig.3). Conductive part (copper or aluminum) with density $\rho_{1}$, nonconductive part with density $\rho_{2}, h$ - the frame depth, $\delta$-section diameter of the conductive frame (axis $\mathrm{OZ}$ oriented in the line of the conductor axis)

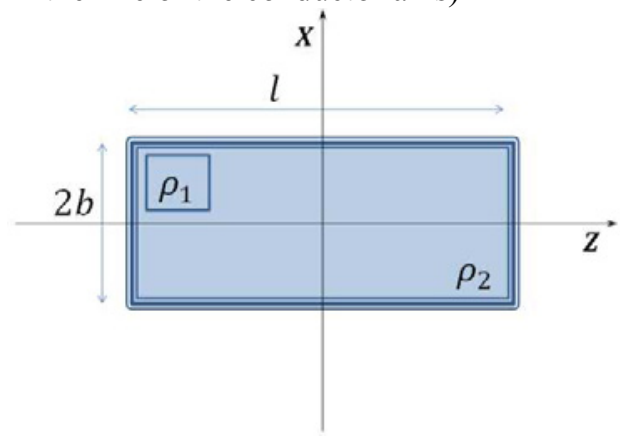

Fig. 3 - The suspended plate parameters

$\overline{{ }^{a} \text { Corresponding author: skubov.dsk } @ y a n d e x . r u ~}$ 

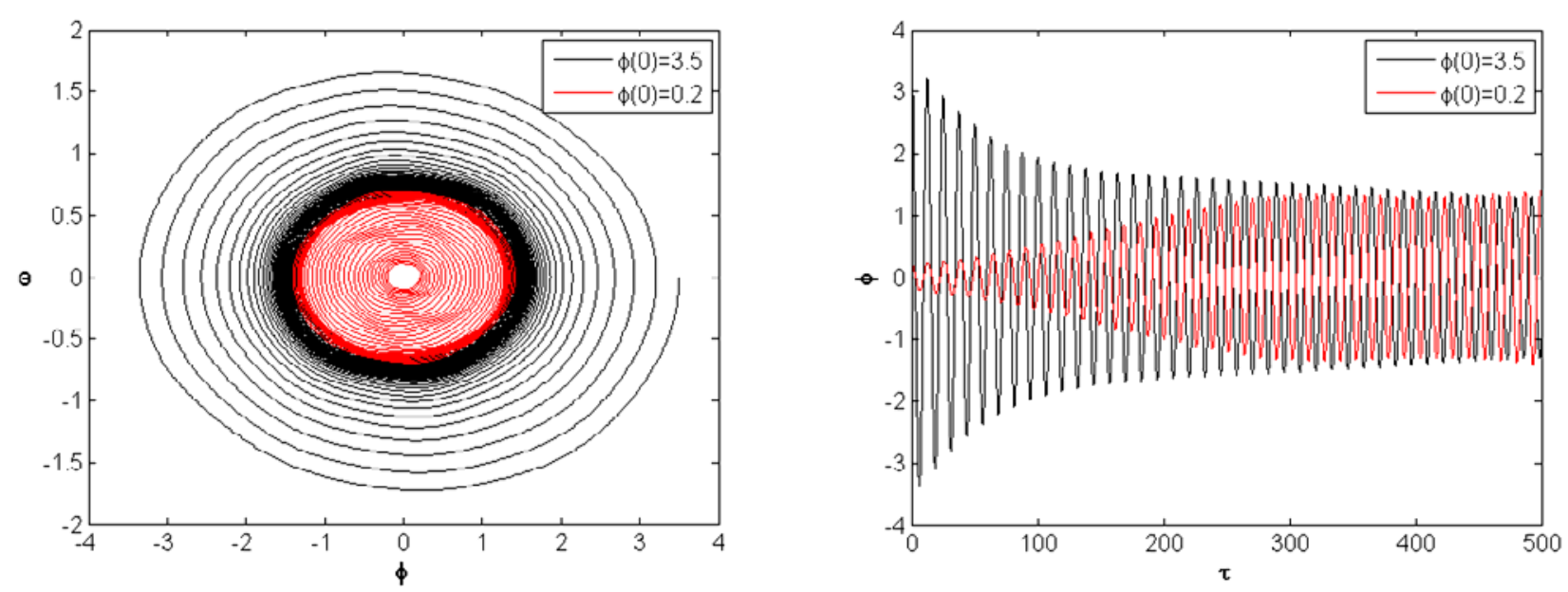

Fig. 4 - Periodic rotary oscillations of system (12)

Electromechanical processes system of differential equations [3] in this case has the following form (11):

$$
\left\{\begin{array}{c}
J \ddot{\varphi}+\beta \dot{\varphi}+c \varphi=-\mu \mu_{0} \frac{i I_{0}(t) a b l \cos \varphi\left[a^{2}+b^{2}\right]}{\left[\left(a^{2}+b^{2}\right)^{2}-4 a^{2} b^{2} \sin ^{2} \varphi\right]}, \\
L(i)+r i= \\
-\mu_{0} \mu \frac{I_{0} l}{2 \pi}\left\{v \cos v t \ln \left[\frac{r_{+}(\varphi)}{r_{-}(\varphi)}\right]+\sin v t \frac{\frac{d r_{+}}{d \varphi} r_{-}-\frac{d r_{-}}{d \varphi} r_{+}}{r_{+} r_{-}} \dot{\varphi}\right\}
\end{array}\right.
$$

where $c$ - the frame base stiffness coefficient under the rotation, $\beta$ - coefficient of linear dissipation, $L$ - selfinductance of the rectangular frame.

Transform system (11) to "dimensionless" form using the parameters:

$$
\begin{gathered}
\tau=v t \rightarrow \frac{\partial}{\partial t}=v \frac{\partial}{\partial \tau}, \quad \tilde{\imath}=\frac{i}{I_{0}}, \quad \frac{c}{J}=\lambda^{2}, \quad \alpha=\frac{2 a b}{a^{2}+b^{2}}, \\
\frac{\beta}{J v}=2 n, \quad r=\frac{R}{L v}, \quad \chi=\mu \mu_{0} \frac{l}{4 \pi L}, \quad \gamma=\mu \mu_{0} \frac{l l_{0}^{2}}{2 \pi J v^{2}}
\end{gathered}
$$

Substitution (11) to form (12)

$$
\left\{\begin{array}{c}
\varphi^{\prime \prime}+2 n \varphi^{\prime}+\frac{\lambda^{2}}{v^{2}} \varphi=-\frac{\gamma \tau \sin \tau(\alpha \cos \varphi)}{1-\alpha^{2} \sin ^{2} \varphi} \\
\tilde{\imath}^{\prime}+r \tilde{\imath}= \\
-\chi\left[\cos \tau \ln \left(1+\frac{2 \alpha \sin \varphi}{1-\alpha \sin \varphi}\right)+\frac{\sin \tau \cos \varphi \alpha}{\sqrt{1-\alpha^{2} \sin ^{2} \varphi}} \varphi^{\prime}\right]
\end{array}\right.
$$

It is a system of nonlinear differential equations with periodic coefficients. For the initial conditions corresponding to a small and large frame deviation from a balance position, in case of real geometrical parameters periodic rotary oscillations have been received (Fig. 4). Left part of Fig.4 represents the phase diagram, right part time dependent oscillations of plate rotation angle.

If coefficients in a second equation of a system (12), which describes change of induced current in a frame, satisfy the natural assumption $r, \chi \gg 1$, it is permissible to neglect a first derivative of current in this equation when receiving a stationary mode of rotation. We get a nonlinear equation with periodic coefficients after substitution of an explicit expression of an induced current, which depends on $\tau, \varphi$ and $\varphi^{\prime}$, to the first equation of a system (12):

$$
\begin{gathered}
\varphi^{\prime \prime}+2 n \varphi^{\prime}+\frac{\lambda^{2}}{v^{2}} \varphi= \\
-\frac{\gamma \chi}{r}\left[\cos \tau \ln \left(1+\frac{2 \alpha \sin \varphi}{1-\alpha \sin \varphi}\right)\right. \\
\left.+\sin \tau \cos \varphi \frac{\alpha}{\sqrt{1-\alpha^{2} \sin ^{2} \varphi}} \varphi^{\prime}\right] . \\
\cdot \sin \tau \frac{\alpha \cos \varphi}{1-\alpha^{2} \sin ^{2} \varphi}
\end{gathered}
$$

The analysis of an equation (13) in assumption of small values of a deviation angle $\varphi$ was performed in order to define the values of parameters, a frame torsional stiffness primarily, which satisfy the conditions of frame parametrical rotary oscillations.

The linearized equation for (13) is the following: $\varphi^{\prime \prime}+2\left(n-q \sin ^{2} \tau\right) \varphi^{\prime}+\left(\Omega^{2}-q \sin 2 \tau\right) \varphi=0$, where $q=\frac{\alpha^{2} \chi \gamma}{r}, \Omega=\frac{\lambda}{v}$.

Numerically evaluated Ince-Strutt diagram for the equation (14) at different values of a dissipation coefficient $n$ is shown in Fig.5.

The analysis of dynamics of multiplicators on a complex plane was performed for the equation (14) by means of varying the parameter $\Omega$ at a constant value of $q=7$. initially, at values of $0<\Omega<2.13$, the two roots of a characteristic polynomial for the equation (14), which are the monodromy matrix $\mathrm{C}$ multiplicators [2]

$$
\lambda^{2}-\operatorname{tr} C \cdot \lambda+\operatorname{det} C=0
$$

are located on a real axis on the different parties of a unit circle. They quickly move towards each other, and after a meeting they "slide" on the circle which radius corresponds to the absolute value of a meeting position, being thus inside a unit circle. Further, at a value of $\Omega \cong 2.7$, one of multiplicators leaves a unit circle again. This case corresponds to the first area of instability in Fig.5. At values of $\Omega>3.5$ the roots return and slide on the circle inside the unit one. The described process is shown in Fig.6.

\footnotetext{
$\overline{{ }^{a} \text { Corresponding author: skubov.dsk } @ y a n d e x . r u ~}$
} 


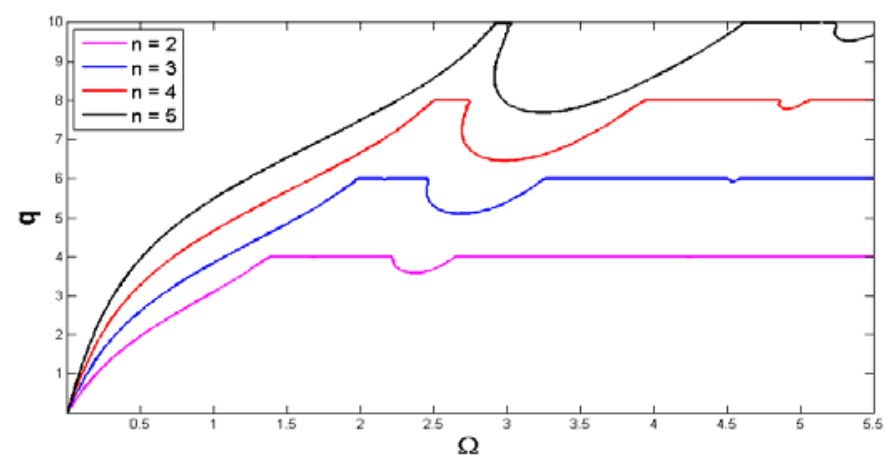

Fig. 5 - Ince-Strutt diagram

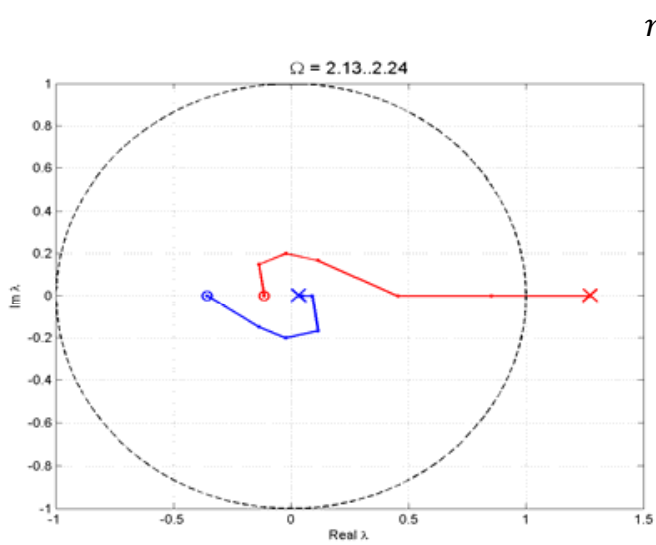

$$
n=4, \quad q=7
$$
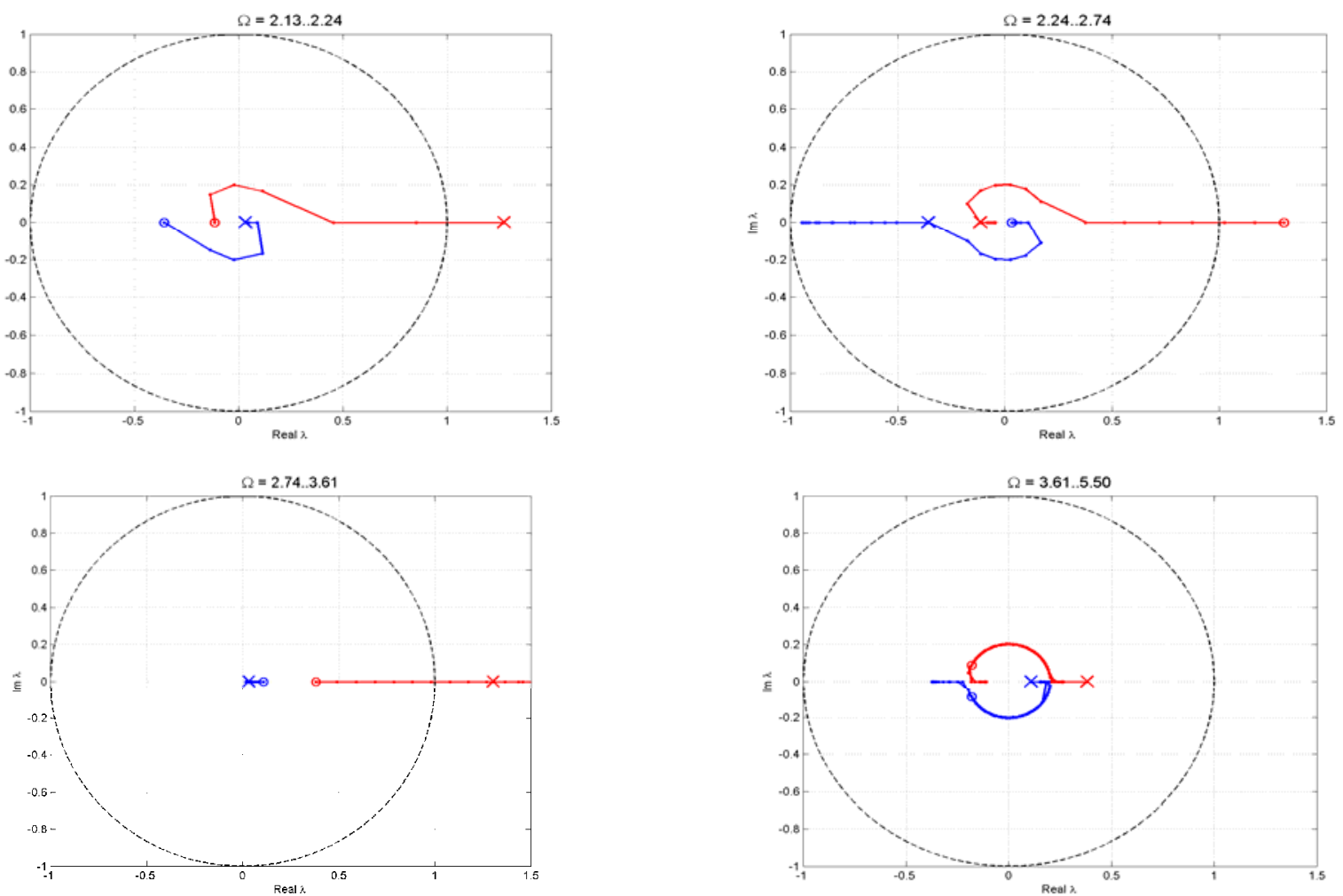

Fig.6 - Dynamics of multiplicators on a complex plane

The fact that contrary to classical Ince-Strutt diagram for Mathieu equation with constant linear dissipation coefficient in this instance we have smooth upper bound for the stability domain in parameter $q$, is an important computational calculation result for linearized equation (13).

The fact, that in any value of parameter $n$ stability domain in parameters space $(\Omega, q)$ is located under the level $q=2 n$ corresponded to zero value of average friction coefficient, is significant. Therefore, we obtain the opportunity to set the system on the resonance oscillations in a large zone of frequency $\Omega$, and this guarantee the efficiency of cooling system during the exploitation and evolution of its mechanical properties.

\section{References}

1. A. P. Aksenov. Mathematics. Mathematical analysis.vol.2 (SPbSTU, SPb, 2005) (in Russian)

2. I. G. Malkin. Theory of motion stability (Nauka, Moscow, 1966) (in Russian)

3. U. I. Neymark, N.A. Fufaev. Dynamics of nonholonomic systems (Nauka, Moscow, 1967) (in Russian)

4. I. E. Tamm. The basis of the theory of electricity (Nauka, Moscow, 1989) (in Russian)

5. Ali. H. Nayfeh. Introduction to Perturbation Techniques (John Wiley \& Sons, Inc, Canada, 1981)

6. Bogolyubov N. N., Mitropolsky Yu.A. Asymptotic methods in theory of nonlinear oscillations (Nauka, Moscow, 1974) (in Russian)

\footnotetext{
$\overline{{ }^{a} \text { Corresponding author: skubov.dsk } @ y a n d e x . r u ~}$
} 


\section{CSNDD 2014}

7. Urabe M. Galerkin method for nonlinear periodic systems, Mechanic, 97 (1996) 